\title{
Spectrum of Andreev Bound States in a Molecule Embedded Inside a Microwave-Excited Superconducting Junction
}

\author{
Jonas Sköldberg, Tomas Löfwander, Vitaly S. Shumeiko, and Mikael Fogelström \\ Deptartment of Microtechnology and Nanoscience - MC2, \\ Chalmers University of Technology, S-412 96 Göteborg, Sweden.
}

(Dated: Physical Review Letters 101, 087002 (2008))

\begin{abstract}
Non-dissipative Josephson current through nanoscale superconducting constrictions is carried by spectroscopically sharp energy states, so-called Andreev bound states. Although theoretically predicted almost 40 years ago, no direct spectroscopic evidence of these Andreev bound states exists to date. We propose a novel type of spectroscopy based on embedding a superconducting constriction, formed by a single-level molecule junction, in a microwave QED cavity environment. In the electron-dressed cavity spectrum we find a polariton excitation at twice the Andreev bound state energy, and a superconducting-phase dependent ac Stark shift of the cavity frequency. Dispersive measurement of this frequency shift can be used for Andreev bound state spectroscopy.
\end{abstract}

Supercurrents through mesoscopic or nanosized Josephson junctions are mainly carried by spectroscopically sharp subgap bound states, so-called Andreev bound states (ABS) 1], as predicted theoretically in Refs. 2, 3. When the weak link is a quantum dot with a single or a few discrete levels supercurrents are also predicted to flow mainly through ABS [4]. ABS come in pairs, one state above and one below the Fermi level. The two ABS of the pair have opposite dispersion with the superconducting phase difference over the junction and carry supercurrent in opposite directions across the junction. These two states form a well-defined two-level system that has been suggested as a qubit 5,6 . Before such a qubit can be realized, experimental detection and characterization of this engineered two-level system should be carried out. To our best knowledge, however, no one has to date reported experiments with direct spectroscopic proof for the existence of Josephson-current carrying ABS [7].

A single-wall carbon nanotube (swCNT) embedded between two superconducting metal leads can support a supercurrent [8, 9, 10, 11, 12, 13]. The electronic energy levels of the swCNT, formed through size quantization, can be tuned in and out of resonance with the lead Fermi levels by gating the swCNT. Such superconducting swCNT transistors [11 can further be switched from a Coulomb blockade regime, to a Kondo regime, to a weakly interacting Fabry-Perot regime by changing a back gate voltage [12. The potential for applications of such swCNT quantum-level junctions was demonstrated through the fabrication and detailed functional control of a nano-SQUID, involving two gated swCNT junctions with controlled on-off states as well as controlled $0-\pi$ SQUID states [13. Similar control has been demonstrated using semiconducting nanowire junctions [14, 15, 16.

We propose a method for direct ABS spectroscopy, based on dispersive measurement of a polaritonic state formed by the ABS strongly coupled to a QED cavity mode. Consider two superconducting leads connected by a molecule with one resonant level, as shown in Figure 1. This setup can be realized in the swCNT transistor by tuning the voltage of the gate electrode [11, 12, 13]. The superconducting proximity effect leads to a split of the resonant level into a pair of ABS, where the ABS level splitting can be controlled by tuning the superconducting phase difference in a SQUID setup. When the gate electrode is coupled to an LC-oscillator, induced quantum fluctuations of the gate potential leads to dressed Andreev bound states. By tuning the ABS into resonance with the cavity, and measuring the shift of the cavity base-frequency, the ABS energy can be observed. The complete ABS energy dispersion with respect to the superconducting phase can be extracted by using a cavity with a variable base-frequency [17, 18, 19, 20, 21. The suggested dispersive method does not involve real interlevel transitions and could be simpler to realize than

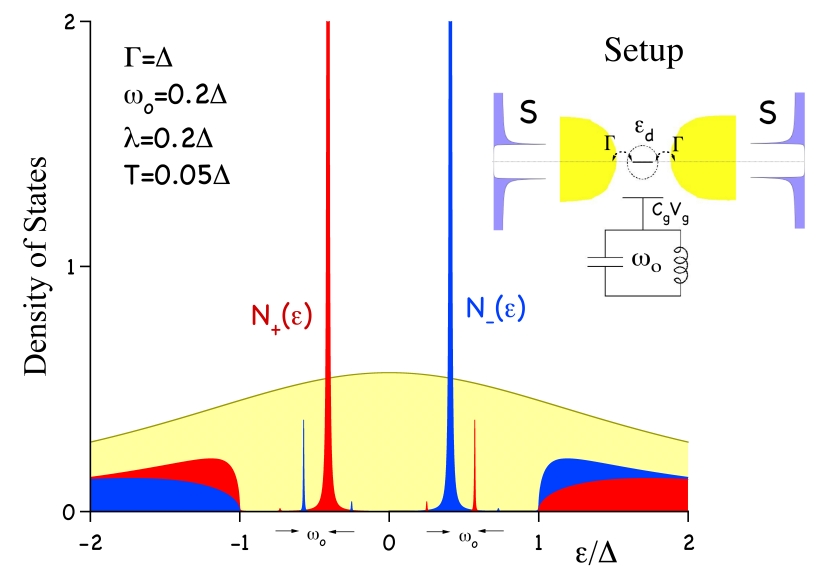

FIG. 1: Superconducting density of states for $\varphi=0.6 \pi$ resolved into right, $N_{+}(\varepsilon)$, and left, $N_{-}(\varepsilon)$, current-carrying branches. The lightly shaded background is the density of states of the resonant level when the contacts are in the normal state. The electron-oscillator coupling leads to dressed Andreev bound states with sidebands separated in energy by the oscillator frequency $\omega_{0}$. The inset shows a sketch of the considered system. 
methods based on microwave absorption [22]. Another advantage of the suggested spectroscopy is that it makes it possible to directly access and characterize individual ABS without measuring dc contact current-voltage characteristics 23. Similar dispersive measurement method has earlier been applied to detect quantum states of superconducting charge qubits 24].

The Hamiltonian for the coupled electron-oscillator system depicted in the inset in Figure 1 is

$$
\hat{H}=\hat{H}_{L}+\hat{H}_{R}+\hat{H}_{\text {level }}+\hat{H}_{T}+\hat{H}_{\text {osc }}+\hat{H}_{\text {level-osc }}
$$

and will be quantified in terms of creation operators for the three excitations of the system: reservoir electrons, $\hat{c}_{k \sigma, \alpha}^{\dagger}$ (reservoir $\alpha=L, R$, momentum $k$, and spin $\sigma$ ); dot electrons, $\hat{d}_{\sigma}^{\dagger}$; and the oscillator mode $\hat{b}^{\dagger}$. The two superconducting reservoirs are described by a standard BCS Hamiltonian $\hat{H}_{\alpha}=\sum_{k \sigma} \xi_{k} \hat{c}_{k \sigma, \alpha}^{\dagger} \hat{c}_{k \sigma, \alpha}+$ $\sum_{k}\left(\Delta_{\alpha} \hat{c}_{k \uparrow, \alpha}^{\dagger} \hat{c}_{-k \downarrow, \alpha}^{\dagger}+\right.$ h.c. $)$, where h.c. denotes hermitian conjugate, $\xi_{k}$ is the quasiparticle dispersion, and $\Delta_{\alpha}=\Delta(T) \mathrm{e}^{i \varphi_{\alpha}}$ are the order parameters of the reservoirs that have the same temperature dependent gap $\Delta(T)$, but have a tunable superconducting phase difference $\varphi=\varphi_{R}-\varphi_{L}$ between them. The reservoirs are coupled by tunneling through a single non-interacting molecular level described by $\hat{H}_{\text {level }}=\sum_{\sigma} \varepsilon_{d} \hat{d}_{\sigma}^{\dagger} \hat{d}_{\sigma}$, where the level energy $\varepsilon_{d}\left(V_{g}\right)$ is tunable by the gate voltage. The lead-to-level tunneling is described by, $\hat{H}_{T}=$ $\sum_{k \sigma, \alpha}\left(v_{k \sigma, \alpha} \hat{c}_{k \sigma, \alpha}^{\dagger} \hat{d}_{\sigma}+\right.$ h.c. $)$. We consider symmetric, spin-independent coupling between the level and the two leads, $v_{k \sigma, L}=v_{k \sigma, R} \equiv v \delta_{k, k_{F}}$, which corresponds to an effective tunneling rate at each barrier $\Gamma=\frac{|v|^{2}}{2 \pi} \mathcal{N}_{F}$, where $\mathcal{N}_{F}$ is the normal state density of states of the leads at the Fermi-level. The oscillator mode with frequency $\omega_{o}$ is described by the Hamiltonian $\hat{H}_{o s c}=\omega_{o} \hat{b}^{\dagger} \hat{b}$. The coupling of the molecular level to the oscillator is described by a linear interaction $\hat{H}_{\text {level-osc }}=\sum_{\sigma} \lambda\left(\hat{b}+\hat{b}^{\dagger}\right) \hat{d}_{\sigma}^{\dagger} \hat{d}_{\sigma}$, where $\lambda$ is the coupling strength. We will assume throughout this paper that the molecular level is aligned with the Fermi levels of the leads, i.e. $\varepsilon_{d}=0$.

We solve the Hamiltonian (1) treating the tunneling to infinite order in the hopping [25, 26, 27] and the electronoscillator coupling perturbatively in a self-consistent Born-approximation [28, 29, 30, 31, 32, 33. The problem reduces to the coupled molecular level/oscillator system. Superconductivity modifies the electronic state on the molecular-level by splitting it into two branches $(s= \pm 1)$ each described by a retarded Green's function

$$
G_{s}^{R}(\varepsilon)=\frac{\bar{\Omega}(\varepsilon)}{z_{s}^{R}(\varepsilon) \varepsilon^{R}+s \Delta \cos (\varphi / 2)},
$$

where the energy-renormalisation factor $z_{s}(\varepsilon)$ is defined by $z_{s}^{R}(\varepsilon) \varepsilon=\tilde{\varepsilon}^{R}+\bar{\Omega}(\varepsilon)\left(\varepsilon^{R}-\Sigma_{-s}^{R}(\varepsilon)\right)$, with $\bar{\Omega}(\varepsilon)=$ $\sqrt{|\Delta|^{2}-\left(\tilde{\varepsilon}^{R}\right)^{2}} / 2 \Gamma$. The state of the oscillator is given by the retarded Green's function

$$
D^{R}(\omega)=\frac{2 \omega_{o}}{\left(\tilde{\omega}^{R}\right)^{2}-\omega_{o}^{2}-2 \omega_{o} \Pi^{R}(\omega)} .
$$

The electron-oscillator coupling enters via the retarded self-energies $\Sigma_{s}^{R}(\varepsilon)$ and $\Pi^{R}(\omega)$ which are determined self-consistently by numerical iteration. $\Sigma_{s}^{R}(\varepsilon)$ and $\Pi^{R}(\omega)$ are functionals of $G_{s}^{R}(\varepsilon)$ and $D^{R}(\omega)$ [and of $G_{s}^{K}(\varepsilon)=-2 i \operatorname{Im} G_{s}^{R}(\varepsilon) n_{e}(\varepsilon)$, with $n_{e}(\varepsilon)=\tanh (\varepsilon / 2 T)$ and $D^{K}(\omega)=-2 i \operatorname{Im} D^{R}(\omega) n_{b}(\omega)$, with $n_{b}(\omega)=$ $\left.\operatorname{coth}(\omega / 2 T), G_{s}^{A}=\left(G_{s}^{R}\right)^{*}\right]$ and defined as

$$
\begin{aligned}
& \Sigma_{s}^{R}(\varepsilon)=i \frac{\lambda^{2}}{2}\left[D^{K}(\omega) \circ G_{s}^{R}(\varepsilon-\omega)+D^{R}(\omega) \circ G_{s}^{K}(\varepsilon-\omega)\right], \quad(4) \\
& \Pi^{R}(\omega)=-i \lambda^{2} \sum_{s= \pm 1}\left[G_{-s}^{R}(\varepsilon) \circ G_{s}^{K}(\varepsilon-\omega)+G_{-s}^{K}(\varepsilon) \circ G_{s}^{A}(\varepsilon-\omega(\text { ) })\right.
\end{aligned}
$$

where the convolution is defined as $a(x) \circ b(x-y)=$ $\int_{-\infty}^{\infty} \frac{d x}{2 \pi} a(x) b(x-y)$. Upon reaching self-consistency the Josephson current is calculated as

$$
I(\varphi)=\frac{e}{\hbar} \Delta \sin \frac{\varphi}{2} \sum_{s= \pm 1} \int_{-\infty}^{\infty} \frac{d \varepsilon}{2 \pi} s \operatorname{Im}\left[\frac{G_{s}^{R}(\varepsilon)}{\bar{\Omega}(\varepsilon)}\right] n_{e}(\varepsilon) .
$$

Inelastic coupling to the environment is introduced phenomenologically above by $\tilde{\epsilon}^{R}=\epsilon^{R}+i \gamma$ and $\tilde{\omega}^{R}=$ $\omega^{R}+i \kappa$. The parameter $\gamma \ll \Delta$ describes a residual phase-breaking scattering rate in the superconducting reservoirs. In absence of an electron-oscillator coupling it is $\gamma$ that limits the life time of the ABS. $\kappa$ describes the finite (long) life time of the mode originating from a finite (but high) quality factor, $Q$, such that $\kappa=\omega_{0} / Q \ll \omega_{0}$. In the present calculations we set $\gamma=\kappa=10^{-3} \Delta$.

Without coupling to the oscillator, the Josephson effect in this system is well known [4. The Green's function-amplitudes, $G_{s}^{R}$, describe two different quasiparticle branches that form on the dot, one having an ABS below $(s=+1)$ and one having an ABS above $(s=-1)$ the Fermi level, see Figure 1. The two branches contribute to the Josephson current (6) in opposite manner. For sub-gap energies $G_{s}^{R}(|\varepsilon|<\Delta)$ contributes to the current in the positive direction for $s=1$ (left to right over the junction) and vice versa for the $s=-1$ branch. The continuum part of $G_{s}^{R}(|\varepsilon|>\Delta)$ contributes to the current in the opposite direction compared with its corresponding sub-gap part.

The electron-oscillator interaction couples the two quasiparticle branches. This is seen explicitly in the energy-renormalization factor $z_{s}$ of one branch which is modified by the self-energy $\Sigma_{-s}^{R}$ of the other branch. In Figure 2 we show a fully self-consistent calculation of the Andreev spectrum as function of superconducting phasedifference. For the chosen parameters there is a resonance between the oscillator and the ABS, i.e. $2 E_{A}(\varphi)=\omega_{o}$, at $\varphi \approx 0.9 \pi$. Away from resonance, the ABS is shifted

$$
E_{A} \rightarrow \bar{E}_{A}=E_{A}+\frac{\lambda^{2} \mathcal{A}_{E}^{2}}{2}\left(\frac{\Phi_{+}}{2 E_{A}+\omega_{o}}+\frac{\Phi_{-}}{2 E_{A}-\omega_{o}}\right)
$$



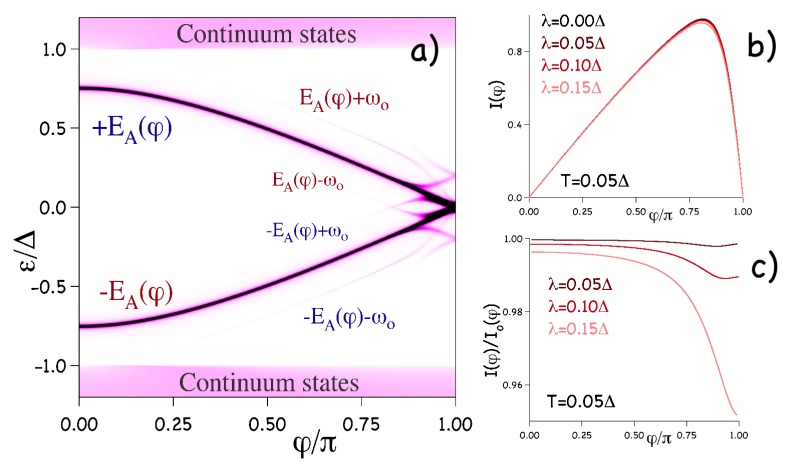

FIG. 2: (a) The spectrum of Andreev-bound states as function of superconducting phase-difference $(\varphi)$ for electronoscillator coupling $\lambda=0.1 \Delta$ and oscillator frequency $\omega_{o}=$ $0.2 \Delta$. The tunneling rate is $\Gamma=\Delta$ and the temperature is $T=0.05 \Delta$. The side bands to each ABS are due to the dressing of the ABS by the electron-oscillator coupling. The sidebands of the ABS with branch index $s= \pm 1$ belong to the quaasiparticle branch with index $s \mp 1$. (b) The currentphase relation for different electron-oscillator couplings for $T=0.05 \Delta$. (c) The reduction of current due to coupling to the oscillator as a function of phase for the same temperature and electron-oscillator couplings as in panel b).

as compared with the case without coupling to the oscillator, but with retained phase-dependent spectral weight $\mathcal{A}_{E}$ of the state at $\varepsilon= \pm E_{A}(\varphi)$. The effective electronoscillator coupling in the sub-gap region is given by the product $\lambda \mathcal{A}_{E}$. In equation (7) thermal occupation factors enter in the combinations $\Phi_{ \pm}=n_{b}\left(\omega_{o}\right) \pm n_{e}\left(E_{A}\right)$. Apart from the shifted ABS we find satellite resonances at $\varepsilon=$ $s \bar{E}_{A} \pm \omega_{o}$ with spectral weights $\frac{1}{2} \lambda^{2} \mathcal{A}_{E}^{3} \Phi_{ \pm s} /\left(2 s E_{A} \pm \omega_{o}\right)^{2}$. It is only at resonance, $2 E_{A}=\omega_{o}$, the satellite on either branch with spectral weight $\propto \Phi_{-}$interfere with the main ABS of the same branch index. This is seen as a precursor of an avoided crossing in Figure 2.

The Josephson current-phase relation is presented in panels b and c of Figure 2 for the case that the electron and oscillator systems are in thermal equilibrium. Due to the different magnitudes of the thermal factors $\Phi_{ \pm}\left(\Phi_{+} \approx 2\right.$ and $\Phi_{-} \approx 0$ for $\left.T \lesssim E_{A}, \omega_{o}\right)$, the current contribution of the satellites is dominated by the satellite with weight $\propto \Phi_{+}$. This satellite reduces the Josephson current by $\sim \lambda^{2} \mathcal{A}_{E}^{2} /\left(2 E_{A}+\omega_{o}\right)^{2}$, which is of the order of a few percent of the full current for our parameter values. There is no dramatic signature in the current-phase relation of an emerging anticrossing at resonance because interference occurs between states carrying current in the same direction, and moreover, these states have spectral weights shared between them drawn from the original ABS, and the population of the states is largely phaseindependent.

The Josephson current through the level modifies the oscillator spectrum giving it a phase-dependence that is shown in Figure 3(a) for the same parameters as for the
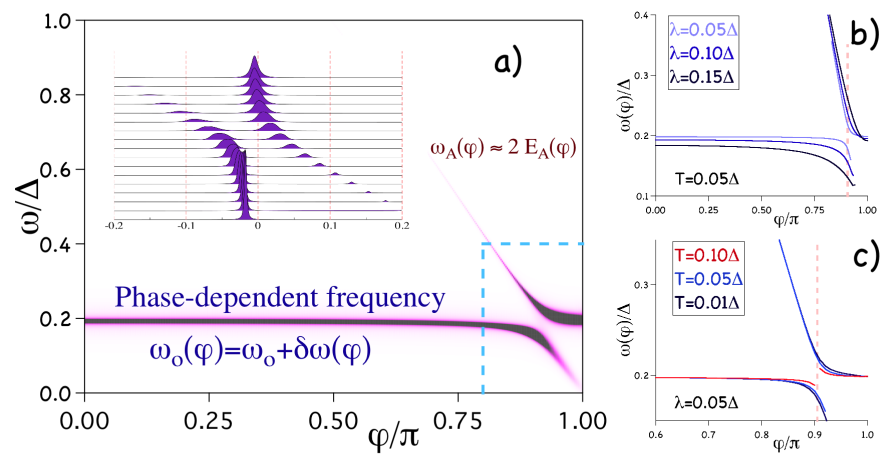

FIG. 3: (a) The spectra of the oscillator plotted as function of superconducting phase-difference with the same parameters as in Figure 2. A mode emerges at $\omega_{A}(\varphi)=2 E_{A}(\varphi)$ and develops to a polariton when in resonance with the oscillator (inset, $0.8 \pi \leq \varphi \leq \pi$ in steps of $0.0125 \pi$ ). The dashed box indicate where the spectra in the inset are taken. Away from resonance there is a Stark shift $\delta \omega(\varphi)$ of the base frequency. (b) The Stark shift is shown at different electron-oscillator coupling strengths for $T=0.05 \Delta$ and (c) at different temperatures for a electron-oscillator coupling strength $\lambda=0.05 \Delta$. In both cases $\omega_{o}=0.2 \Delta$. The dashed line in panels b)-c) indicate the resonant phase.

ABS spectrum in Figure2 The polarization $\Pi^{R}(\omega)$ gives the possible collective excitations that are supported by the electronic system. Iterating the self-consistency equations once we get

$$
\Pi^{R}(\omega)=2 \lambda^{2} \frac{\mathcal{A}_{E}^{2}}{\left(\omega^{R}\right)^{2}-4 E_{A}^{2}} n_{e}\left(E_{A}\right)
$$

for the retarded phonon self-energy. As may be expected there is a mode with the phase-dispersion $\omega_{A}=2 E_{A}(\varphi)$ originating from the transitions between the two ABS and the subsequent emission or absorption of the energy $\omega_{A}$. A spectral weight $\sim 2 \lambda^{2} \mathcal{A}_{E}^{2}\left[4 \omega_{o}^{2} /\left(4 E_{A}^{2}-\omega^{2}\right)^{2}\right] n_{e}\left(E_{A}\right)$ for this collective excitation is vanishingly small away from resonance. Near resonance the oscillator mode and the excitation interact strongly and an avoided crossing appears with a frequency split at $2 E_{A}=\omega_{o}$

$$
\omega_{o}(\varphi)=\omega_{o}+\frac{\delta}{2} \pm \sqrt{\left(\frac{\delta}{2}\right)^{2}+2 \lambda^{2} \mathcal{A}_{E}^{2} n_{e}\left(E_{A}\right)}
$$

where $\delta=2 E_{A}-\omega_{o}$ is the detuning. The analytic estimate of the split, $2 \lambda \mathcal{A}_{E} \sqrt{2 n_{e}\left(E_{A}\right)} \approx 0.096$, for a nearly phase-independent $\mathcal{A}_{E} \sim 0.34$ when $\Gamma=\Delta$, is in good agreement with the self-consistently determined split, $\approx 0.1 \Delta$, extracted from the inset in Figure 3(a) at $\varphi=0.9 \pi$. It is important to note that this split is significantly larger than the intrinsic broadening of the spectral features $(\sim \kappa)$ and signals a strong-coupling regime in the sense of cavity-QED, i.e. $\lambda \mathcal{A}_{E} \gg \kappa$.

Away from resonance, the electron-oscillator coupling gives a negative phase-dependent Stark shift of the oscillator base frequency. We plot this shift in Figure 3(b)-(c) 
both as a function of electron-oscillator coupling strength $\lambda$ for $T=0.05 \Delta$ and as a function of temperature for $\lambda=0.05 \Delta$. Our analytic estimate in equation $(9)$ gives in the limit of large detuning

$$
\omega_{o}(\varphi)=\omega_{o}+\delta \omega(\varphi)=\omega_{o}-2 \frac{\lambda^{2} \mathcal{A}_{E}^{2} n_{e}\left(E_{A}\right)}{2 E_{A}-\omega_{o}}
$$

This analytical expression for $\delta \omega(\varphi)$ is a good approximation when the continuum contributions can be neglected, i.e. for $\Gamma / \Delta \ll 1$, while in general one must use numerics to extract $\delta \omega(\varphi)$. Dispersive measurement of the acquired phase-dependent resonance frequency of the oscillator, $\omega_{o}(\varphi)$, gives a possibility to detect the position of the polariton resonance and hence define the energy of the ABS. Furthermore, by sweeping the base frequency of the oscillator the phase dispersion of the ABS energy can be detected.

Assume that we bridge two aluminium superconductors by a gated swCNT, as shown in the inset of Figure 1 . The gate is part of an LC-circuit which leads to an oscillation of the gate voltage with frequency $\omega_{o}=1 / \sqrt{L C}$. A practical value for the resonator frequency is $\sim 10 \mathrm{GHz}$, for which the polariton resonance is located well inside the superconducting gap (for aluminium $\Delta_{\mathrm{Al}} \approx 50 \mathrm{GHz}$ ), and at the same time the ABS energy splitting is large compared to the temperature below $100 \mathrm{mK}$. In our calculations we neglect electron-electron interactions. This can be done if $\Gamma$ is large compared to $\Delta$, which can experimentally be realized by tuning $\Gamma$ by a back gate 12 to approach the weakly interacting Fabry-Perot regime. The main modification of the one-iteration approximation in the case $\Gamma \gg \Delta$ is that $\mathcal{A}_{E}$ becomes strongly phase dependent, tending to $(\Delta / 2 \Gamma) \sin (\varphi / 2)$ as $\Gamma / \Delta$ grows. This gives the effective coupling $\lambda \mathcal{A}_{E} \sim \lambda \Delta / \Gamma$ close to the resonance. This coupling must be large compared to the intrinsic oscillator damping, $\lambda \Delta / \Gamma \gg \kappa=\omega_{o} / Q$, in order to resolve $\delta \omega(\varphi)$. The strength of the bare coupling $\lambda$ is determined by the capacitive interaction between the gate and the dot, and it is proportional to the ratio of corresponding capacitances, $C_{g} / C_{\Sigma}$, and can be expressed through the oscillator frequency as $\lambda=\left(C_{g} / C_{\Sigma}\right)\left(E_{C} / 8 E_{L}\right)^{1 / 4} \omega_{o}\left(E_{C}\right.$ and $E_{L}$ are charging and inductive energies of the oscillator, respectively). For swCNT superconducting contacts the gate capacitance can be comparable with the capacitances of the contacts to the leads, having values of tens of aF 34. Thus the coupling $\lambda$ can be on the order of $10 \%$ of the oscillator frequency as assumed in our calculation. When the gate of the contact is connected to a superconducting cavity, the quality factor of the oscillator can be of order 1000 - 10000 [17, 18, 19, 20]. Given our calculated value for effective ABS-oscillator coupling, $\lambda \mathcal{A}_{E}$, we get the ratio $\lambda \mathcal{A}_{E} / \kappa \approx 100-1000$, indicating that the strong coupling regime is indeed feasible, the resolution of the proposed spectroscopy should be very favorable to encourage experiments.
We acknowledge valuable discussions with V. Bouchiat, P. Delsing, D. Feinberg, K. Grove-Rasmussen, P. Hyldgaard, G. Johansson, H. I. Jørgensen, G. Wendin, C. Wilson, and A Zazunov. The research was supported by the Swedish Research Council (VR) and the Swedish Foundation for Strategic Research (SSF), and the EC FP7 program under grant agreement "SINGLE" No. 213609 .

[1] Andreev, A. F. Zh.Eksp.Teor.Fiz. 49, 665 (1965)

[2] Kulik, I. O. Zh.Eksp.Teor.Fiz. 57, 1745 (1969)

[3] Ishii, C. Prog. Theor. Phys. 44, 1525 (1970)

[4] Beenakker, C. W. J. \& van Houten, H. In Singleelectron tunneling and mesoscopic devices. Eds. H. Koch \& H. Lübbig, pp 175-179, Springer, Berlin, (1992)

[5] Zazunov, A. et al Phys. Rev. Lett. 90, 087003 (2003)

[6] Zazunov, A. et al Phys. Rev. B. 71, 214505 (2005)

[7] The related vortex core states have been verified by scanning tunneling microscopy: Hess, H. F. et al Phys. Rev. Lett. 62, 214 (1989)

[8] Kazumov, A. Yu. et al. Science 284, 1508 (1999)

[9] Buitelaar, M. R. et al. Phys. Rev. Lett. 89, 256801 (2002)

[10] Buitelaar, M. R. et al. Phys. Rev. Lett. 91, 057005 (2003)

[11] Jarillo-Herrero, P., van Dam, J. A., \& Kouwenhoven, L. P. Nature 439, 953 (2006)

[12] Jørgensen, H. I. et al. Phys. Rev. Lett. 96, 207003 (2006)

[13] Cleuziou, J. P. et al. Nature Nanotech. 1, 53 (2006)

[14] van Dam, J. A., et al. Nature 442, 667 (2006)

[15] Xiang, J. et al, Nature Nanotech. 1208 (2006)

[16] Marchenkov, A. et al. Nature Nanotech. 2, 481 (2007)

[17] K. D. Osborn, J. A. Strong, A. J. Sirois, \& R. W. Simmonds, IEEE Trans. Appl. Supercond 17, 166 (2007)

[18] M. A. Castellanos-Beltran \& K. W. Lehnert, Appl. Phys. Lett., 91, 083509 (2007)

[19] A. Palacios-Laloy, et al, J. Low Temp. Phys. 1511034 (2008)

[20] Sandberg, M et al, Appl. Phys. Lett. 92, 203501 (2008)

[21] Wallquist, M., Shumeiko, V.S., \& Wendin, G., Phys. Rev. $B$ 74, 224506 (2006)

[22] Gorelik, L. Y. et al. Phys. Rev. Lett. 75, 1162 (1995)

[23] Della Rocca, M. L. et al. Phys. Rev. Lett. 99, 127005 (2007).

[24] Wallraff, A. et al. Nature 431, 165 (2004).

[25] Jauho, A. P., Wingreen, N. S. \& Y. Meir, Y., Phys. Rev. $B$ 50, 5528 (1994)

[26] Cuevas, J. C., Martín-Rodero, A., \& Levy Yeyati, A. Phys. Rev. B 54, 7366 (1996)

[27] Cuevas, J. C. \& Fogelström, M. Phys. Rev. B 64, 104502 (2001)

[28] Hyldgaard, P. et al Ann. Phys. 236, 1 (1994)

[29] Mitra, A., Aleiner, I., \& Millis, A. J. Phys. Rev. B 69, $245302(2004)$

[30] Viljas, J. et al Phys. Rev. B 72, 245415 (2005)

[31] Novotný, T., Rossini, A., \& Flensberg, K. Phys. Rev. B 72, 224502 (2005)

[32] Zazunov, A., Egger, R., Mora, C., \& Martin, T. Phys. Rev. B 73, 214501 (2006)

[33] Zazunov, A., Feinberg, D., \& Martin, T. Phys. Rev. Lett. 
97, 196801 (2006)

Physica E 40, 92 (2007)

[34] Grove-Rasmussen, K., Jørgensen, H. I., \& Lindelof, P. E. 